Andrews University

Digital Commons @ Andrews University

Faculty Publications

8-2019

\title{
Electron Energization by Parallel Electric Fields in Poloidal Standing Waves
}

\author{
P. A. Damiano \\ University of Alaska, Fairbanks \\ Eun-Hwa Kim \\ Princeton University \\ Jay R. Johnson \\ Andrews University, jrj@andrews.edu \\ P. Porazik \\ Lawrence Livermore National Laboratory
}

Follow this and additional works at: https://digitalcommons.andrews.edu/pubs

Part of the Astrophysics and Astronomy Commons

\section{Recommended Citation}

Damiano, P. A., Kim, E.-H., Johnson, J. R., \& Porazik, P. (2019). Electron energization by parallel electric fields in poloidal standing waves. Journal of Geophysical Research: Space Physics, 124, 6691- 6700. https://doi.org/10.1029/2019JA026849

This Article is brought to you for free and open access by Digital Commons @ Andrews University. It has been accepted for inclusion in Faculty Publications by an authorized administrator of Digital Commons @ Andrews University. For more information, please contact repository@andrews.edu. 


\section{JGR Space Physics}

\section{RESEARCH ARTICLE \\ 10.1029/2019JA026849 \\ Electron Energization by Parallel Electric Fields in Poloidal Standing Waves}

Key Points:

- The first full flux tube kinetic simulations of electron energization in poloidal standing modes are presented

- Electron energization and wave energy dissipation are increased with electron temperature and azimuthal mode number

- The monoenergetic nature of the electron energization is consistent with previously published results for toriodal standing modes

Correspondence to:

P. A. Damiano,

padamiano@alaska.edu

Citation:

Damiano, P. A., Kim, E.-H., Johnson, J. R., \& Porazik, P. (2019). Electron energization by parallel electric fields in poloidal standing waves. Journal of Geophysical Research: Space Physics, 124, 6691-6700. https://doi.org/10.1029/ 2019JA026849

Received 15 APR 2019

Accepted 23 JUL 2019

Accepted article online 31 JUL 2019

Published online 13 AUG 2019

C2019. American Geophysical Union. All Rights Reserved.

\author{
P. A. Damiano ${ }^{1}(\mathbb{D})$, E.-H. Kim $^{2}$ (D) J. R. Johnson ${ }^{3}$, and P. Porazik ${ }^{4}$ \\ ${ }^{1}$ Geophysical Institute, University of Alaska Fairbanks, Fairbanks, AK, USA, ${ }^{2}$ Princeton Plasma Physics Laboratory, \\ Princeton University, Princeton, NJ, USA, ${ }^{3}$ Department of Engineering and Computer Science, Andrews University, \\ Berrien Springs, MI, USA, ${ }^{4}$ Lawrence Livermore National Laboratory,Livermore, CA, USA
}

\begin{abstract}
A hybrid gyrofluid-kinetic electron model is adapted and used to simulate poloidal standing modes for different electron temperatures and azimuthal mode numbers. As in previous studies of toroidal standing modes, mirror force effects lead to increased parallel potential drops, monoenergetic electron energization, and wave energy dissipation as the ambient electron temperature is increased. A similar trend is also observed when the electron temperature is held fixed and the azimuthal mode number increased-owing to the narrowing of the azimuthal flux tube width, which necessitates more electron energization to carry the increased parallel current density. In both cases, the increase in electron energization eventually leads to more rapid decreases in the parallel current with time because of the dissipation of wave energy.
\end{abstract}

\section{Introduction}

Standing waves in the magnetosphere occur in both toroidal and poloidal polarizations (where the perpendicular electric field is in the radial and azimuthal direction, respectively). Toroidal modes generally result from mode conversion from fast compressional modes (Chen \& Hasegawa, 1974; Southwood, 1974), while the generation of poloidal modes are associated with wave-particle interactions in the inner magnetosphere (Chen \& Hasegawa, 1988; Southwood, 1976). Poloidal modes also exist with or without significant magnetic compressional components (e.g., Dai et al., 2015), and both limits have similar occurrence rates that increase during storm time conditions. Although the two polarizations of the standing waves can have different driving mechanisms, these waves are generally neither purely toroidal nor poloidal, but a mixture. Additionally, dominantly poloidal modes can gradually evolve into dominantly toroidal modes due to phase mixing effects (Leonovich \& Mazur, 1998; Mann \& Wright, 1995; Mann et al., 1997; Radoski, 1967) and under certain conditions, poloidal modes may change polarization to toroidal as they propagate across magnetic shells (Klimushkin et al., 2004; Leonovich \& Mazur, 1993).

In the auroral context, toroidal modes have been linked to the formation of some discrete auroral arcs (e.g., Lotko et al., 1998; Samson et al., 2003) and previous kinetic simulations (Damiano \& Wright, 2008; Damiano \& Johnson, 2012) illustrate a monoenergetic energization (consistent with the nature of the observed auroral arcs) under the influence of the wave parallel electric field. In the inner magnetosphere, the dominant azimuthal electric and magnetic fields (for poloidal and toroidal modes, respectively) are in the direction of the magnetospheric particle drift and electrons can be energized by drift/drift-bounce resonance to very high energies (e.g., Elkington et al., 1999; 2003; Ozeke \& Mann, 2008; Shprits et al., 2008), which leads to an inward radial diffusion after multiple wave-particle interactions (e.g., Elkington et al., 2003; Ozeke et al., 2012; Shprits et al., 2008). Modes with high azimuthal wave numbers ( $m \approx 40-120$ ) cannot exhibit resonance with the electron drift motion, but nonresonant interaction of electrons with the wave azimuthal electric field can also lead to rapid radial transport (Ukhorskiy et al., 2009). The acceleration of electrons under the influence of the azimuthal electric field, however, does not preclude the potential for electron energization in poloidal Alfvén modes by the parallel electric fields inherent in the wave (as evident in the toroidal case). For example, in the theory of kinetic ballooning modes (e.g., Cheng \& Lui, 1998; Cheng, 2004), which have poloidal polarization and have been linked to observations of auroral beads (e.g., Donovan et al., 2006; Motoba et al., 2012; Rae et al., 2009), parallel electric fields lead to the acceleration of the electrons. In the magnetohydrodynamic limit, these ballooning modes have zero frequency so we expect there would be 

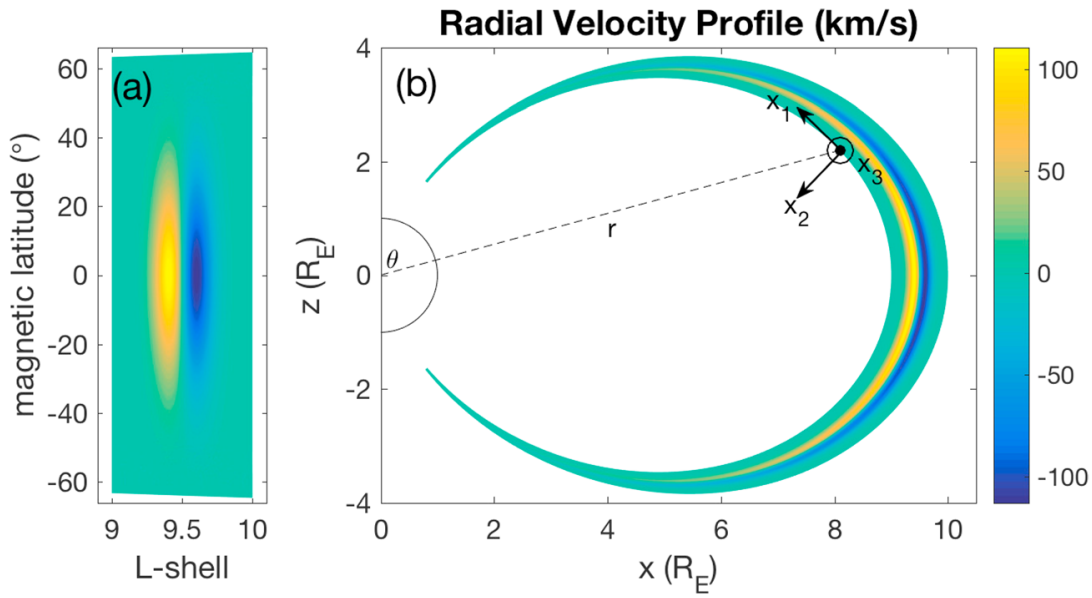

Figure 1. (a) Initial radial velocity perturbation $(\mathrm{km} / \mathrm{s})$ presented as a function of magnetic latitude and L shell. (b) Initial perturbation in cartesian coordinates. Inset displays the relation between polar and curvilinear coordinates where $x_{1}=\cos \theta / r^{2}, x_{2}=\sin ^{2} \theta / r, x_{3}=\phi$, and $\theta$ is the angle subtended from the $z$ axis. The circle of radius $1 R_{E}$ denotes the surface of the Earth. The low-altitude simulation boundary is at a geocentric distance of $2 R_{E}$.

good correspondence between the nature of electron energization in the growth phase of a low-frequency poloidal standing wave and electron energization in a ballooning mode. We have therefore adapted the 2-D gyrofluid-kinetic electron model (Damiano et al., 2007; 2015), previously used to consider toroidal standing (e.g., Damiano \& Wright, 2008; Damiano \& Johnson, 2012) and propagating (Damiano et al., 2015, 2016) modes to study electron energization by parallel electric fields in poloidal standing waves. While this study is interesting in itself, this work also serves as a stepping stone to a more complicated investigation of electron energization in ballooning modes to be conducted in future. The remainder of the paper is divided into three sections. Section 2 presents an overview of the hybrid gyrofluid-kinetic electron model that will be used in the investigation. Section 3 presents the simulation results, while section 4 draws conclusions based on this analysis.

\section{Hybrid Model}

The model used is a version of the 2-D hybrid gyrofluid-kinetic electron model in dipolar coordinates (Damiano et al., 2007, 2015) modified for the inclusion of poloidal Alfvén modes, but for the moment neglecting coupling to the compressional mode. The geometry is illustrated in Figure $1 \mathrm{~b}$ and explicitly includes the field aligned direction $\left(x_{1}\right)$ and the direction across $\mathrm{L}$ shells $\left(x_{2}\right)$. For consideration of the poloidal modes, the gyrofluid portion of the model incorporates the modified linearized radial momentum equation given by

$$
\mu_{o} \rho_{o} \frac{\partial \tilde{u_{2}}}{\partial t}=\frac{B_{o}}{h_{1} h_{2}}\left(\frac{\partial}{\partial x_{1}}\left(h_{2} b_{2}\right)\right),
$$

where $\tilde{u}_{2}=\left(1-1.25 \rho_{i}^{2} \nabla_{\perp}^{2}\right) u_{2}, \rho_{i}$ is the ion gyroradius, $x_{1}=\cos \theta / r^{2}, x_{2}=\sin ^{2} \theta / r, x_{3}=\phi, h_{1}=$ $r^{3} /\left(1+3 \cos ^{2} \theta\right)^{1 / 2}, h_{2}=r^{2} /\left(\sin \theta\left(1+3 \cos ^{2} \theta\right)^{1 / 2}\right)$, and $h_{3}=r \sin \theta$ (where $\theta$ is the angle subtended from the $z$ axis as illustrated in Figure 1 ) and $\rho_{o}$ and $B_{o}$ denote the background plasma density and magnetic field, respectively. The modified fluid velocity $\tilde{u}_{2}$ is defined to include the original cold plasma fluid velocity as well as a gyroviscosity term resulting from an off-diagonal term in the divergence of the pressure tensor. The coefficient of $\rho_{i}^{2} \nabla_{\perp}^{2}$ is obtained from a Padé approximation (Cheng \& Johnson, 1999; Johnson \& Cheng, 1997). Equation (1) is coupled to the radial component of Faraday's law given by

$$
\frac{\partial b_{2}}{\partial t}=\frac{1}{h_{1} h_{3}}\left(-\frac{\partial}{\partial x_{3}}\left(h_{1} E_{1}\right)+\frac{\partial}{\partial x_{1}}\left(h_{3} E_{3}\right)\right)=\frac{1}{h_{1} h_{3}}\left(m h_{1} E_{1}+\frac{\partial}{\partial x_{1}}\left(h_{3} E_{3}\right)\right)
$$


where $m$ is the imposed azimuthal mode number (such that $u_{2}, b_{2}, E_{3} \propto \sin (m \phi)$ and $E_{1}=E_{\|} \propto \cos (m \phi)$ ) and the azimuthal (where a Padé approximation has also been used to relate $\tilde{u}$ and the $\vec{E} \times \vec{B}$ velocity)

$$
E_{3}=B_{o}\left(1-\rho_{i}^{2} \nabla_{\perp}^{2}\right) \tilde{u}_{2}
$$

and parallel Ohm's laws

$$
\begin{aligned}
-m^{2}\left(\frac{h_{2}}{h_{1} h_{3}}+\frac{1}{h_{1} \lambda_{e}^{2}}\right) h_{1} E_{1} & =m\left(\frac{h_{2}}{h_{1} h_{3}} \frac{\partial}{\partial x_{1}}\left(h_{3} E_{3}\right)\right) \\
& +e \mu_{o} \frac{\partial}{\partial x_{1}} \int v_{1}^{2} f \mathrm{~d}^{3} v \\
& +\mu_{o} \frac{e}{m_{e}} \frac{\partial B_{o}}{\partial x_{1}} \int \mu_{m} f \mathrm{~d}^{3} v \\
& -2 \mu_{o} \frac{e}{m_{e}} \frac{\partial B_{o}}{\partial x_{1}} \int \frac{m_{e} v_{1}^{2}}{2 B_{o}} f \mathrm{~d}^{3} v
\end{aligned}
$$

Here, $\mu_{m}=m_{e} v_{\perp}^{2} /(2 B)$ is the electron magnetic moment, the second term on the right-hand side is associated with the parallel gradient of the electron pressure, while the third and fourth terms are associated with the magnetic mirror force incorporating the perpendicular and parallel electron pressures, respectively. This equation was derived, as presented for the toroidal mode in Damiano et al. (2007), using Ampere's law, Faraday's law, and the gyroaveraged Vlasov equation, with the exception that the azimuthal component of Faraday's law $\left(E_{3}\right)$ was used in place of the radial component $\left(E_{2}\right)$ in order to consider poloidal modes.

The model equations are solved using a predictor-corrector method, and equation (4) provides an estimate of parallel electric field at the predictor step. In order to enforce quasineutrality, any residual $\nabla \cdot \vec{j}$, is corrected for using the auxilliary Poisson's equation:

$$
\begin{aligned}
\epsilon_{o} \frac{\partial}{\partial t}\left(\frac{1}{h_{1} h_{2} h_{3}}\left(m h_{1} h_{2} E_{3 c}\right)\right) & =-\nabla \cdot \vec{j} \\
& =\frac{-1}{h_{1} h_{2} h_{3}}\left(\frac{\partial}{\partial x_{1}}\left(h_{2} h_{3} j_{e}\right)+m h_{1} h_{2} j_{3}\right)
\end{aligned}
$$

where $j_{e}=-e \int v_{1} f \mathrm{~d}^{3} v$ is the electron parallel current and $E_{3 c}$ is the correction to the perpendicular electric field. Equation (5) is derived by taking the divergence of the full Ampere's law and noting that $\nabla \cdot \vec{E}_{||} \ll \nabla \cdot \vec{E}_{\perp}$ (e.g., Damiano et al., 2005). The correction is then applied to $E_{\|}$by incorporating $E_{3 c}$ in the first term on the right-hand side of equation (4) at the corrector step (e.g., Damiano et al., 2003; 2007).

The guiding center equations are used to describe the parallel electron dynamics:

$$
\begin{gathered}
m_{e} \frac{\mathrm{d} v_{1}}{\mathrm{~d} t}=-e E_{1}-\mu_{m} \frac{1}{h_{1}} \frac{\partial B_{o}}{\partial x_{1}} \\
h_{1} \frac{\mathrm{d} x_{1}}{\mathrm{~d} t}=v_{1},
\end{gathered}
$$

where $v_{1}$ is the parallel electron velocity and $v_{\perp}=\sqrt{v_{2}^{2}+v_{3}^{2}}$ is the gyrophase independent perpendicular velocity. The corresponding integral moments of the electron distribution functions in equations (4) and (5) are treated computationally as summations using standard particle-in-cell techniques (Damiano et al., 2007).

The neglect of the compressional mode, via the absence of the parallel magnetic field perturbation $\left(b_{1}\right)$, precludes coupling between the toroidal and poloidal Alfvén modes within the simulation and neglects electron energization associated with the parallel magnetic field perturbation (e.g., Klimushkin \& Mager, 2014). For the moment, the model is run independently for consideration of either toroidal or poloidal modes. As mentioned earlier, poloidal modes exist with or without significant compressional components (e.g., Dai et al., 2015) and it is in limit of the latter (which are also generally high $m$ modes) that this work most applies. However, it is our intention to generalize the model in future to include all three components of the magnetic field perturbation in tandem so that it is valid in high $\beta$ plasmas where compressional effects are largest (e.g., Xia et al., 2017). This inclusion is already done in our box model (Damiano et al., 2003). Compressional modes have additionally been considered using both analytical gyrokinetic (Crabtree et al., 2003; Crabtree \& Chen, 2004; Mager et al., 2013) and computational gyrokinetic (Porazik \& Lin, 2011a, 2011b) frameworks. 
(a)

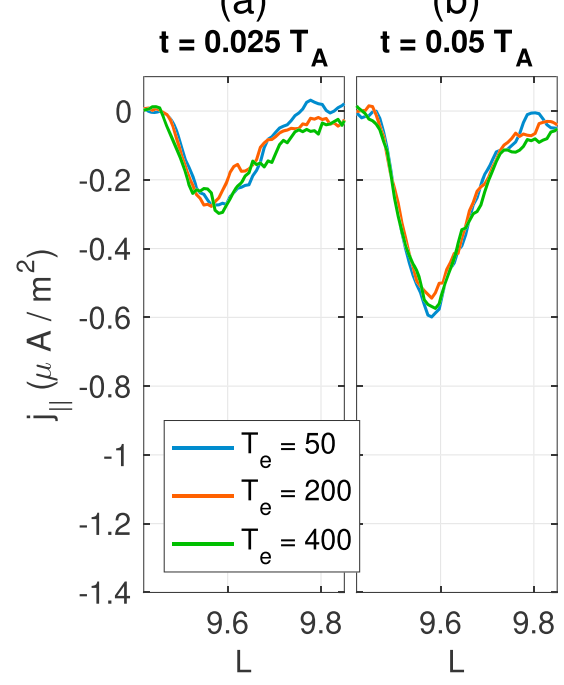

(c) $t=0.1 \mathrm{~T}$

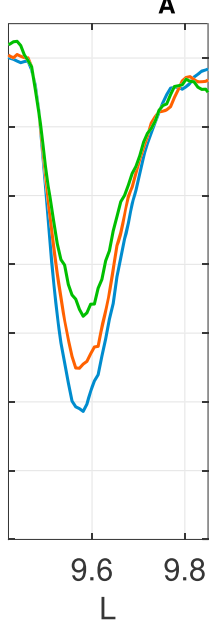

(d) $t=0.15 \mathrm{~T}_{\mathrm{A}} \quad \mathrm{t}=0.2 \mathrm{~T}_{\mathrm{A}}$ ( $\begin{array}{rr}9.6 & 9.8 \\ \mathrm{~L} & \end{array}$ (e) $\mathbf{t}=0.2 \mathrm{~T}_{\mathrm{A}}$

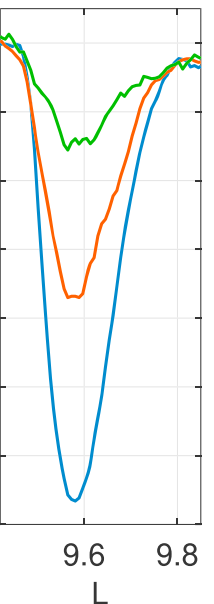

Figure 2. Parallel current evolution above the northern ionospheric boundary for $m=250$ at the indicated times and temperatures.

\section{Simulations}

The simulation is initialized using an eigenmode solution for the velocity perturbation of a fundamental standing mode derived from the model of Taylor and Walker (1984). The exact same functional dependence for the eigenmode as was used in previous studies (e.g., Damiano et al., 2007; Damiano \& Wright, 2008; Damiano \& Johnson, 2012) is used here except that we are initializing the radial (as opposed to azimuthal) velocity in order to drive the poloidal mode. The initial perturbation is displayed in Figure 1 and remains consistent for all the simulations presented. For the present study, we do not consider ion gyroradius effects (assuming $T_{i}=0$ so that $\rho_{i}$ corrections in $\tilde{u}_{2}$ and $E_{2}$ are neglected). This simplification is done for consistent comparison with our previously published works on toroidal standing modes, and $\rho_{i}$ effects will be addressed in a separate publication. In this paper, two limiting cases are considered.

\subsection{Case of Fixed Azimuthal Mode Number and Variable $T_{e}$}

For the present section, we fix the azimuthal mode number to be $m=250$ but vary the electron temperature between 50 and $400 \mathrm{eV}$. Our choice of azimuthal wave number is admittedly high but not unreasonable as cases of $m \sim 200$ have been inferred from observations (e.g., Takahashi et al., 2013) and high $m$ modes do not generally have strong magnetic compressional components (e.g., Dai et al., 2015). We also consider a large range of $m$ values here (more clearly evident in the next section) to emphasize trends. Figure 2 then illustrates the resulting profile of the parallel current for a cut along the northern low-altitude boundary (which is set at a geocentric distance of $2 R_{E}$ ). Three different electron temperature cases are presented, and although the evolution looks quite similar at earlier times, there is a pronounced divergence as time increases. The amplitude of the higher temperature cases $\left(T_{e}=200\right.$ and $\left.400 \mathrm{eV}\right)$ begins to decrease after about $t=0.1-0.15 T_{A}$, while the coldest temperature $\left(T_{e}=50 \mathrm{eV}\right)$ case continues to grow, obtaining the largest amplitude.

Figure 3 then presents the field-aligned parallel current profile along $L=9.6$ and at $t=0.15 T_{A}$ (same time as in Figure 2a). The increased current in all three temperature cases results from the converging magnetic field topology, and the figure illustrates that the diverging current magnitudes evident in Figure 2 extend all along the field line. In order to understand these different parallel current magnitudes as a function of electron temperature, it is necessary to consider the evolution of the electron distribution in each of the cases. Figure 4 plots electron distribution functions at the northern low-altitude boundary in all the temperature cases. The distributions are taken along the field line of maximum parallel current $(L=9.6)$ and are identical in feature to those generated for toroidal standing modes (and presented in Damiano \& Wright, 2008). The distributions are the result of the field-aligned acceleration of electrons (positive displacement to carry the negative field-aligned current) some of which then undergo reflection in the converging magnetic field 




Figure 3. Parallel current density as a function of magnetic latitude along $L=9.6$ field line for indicated temperatures. In all cases, $m=250$. topology and travel back up the field line forming the plotted ring distributions in velocity space. The radius of the ring is indicative of the magnitude of the parallel potential drop in the wave, which from a simple conservation of energy argument results as

$$
e \Delta \phi=\frac{1}{2} m_{e}\left(v_{\|}^{2}+v_{\perp}^{2}\right) .
$$

This relationship is what is expected to occur since the potential drop needed to overcome mirror force repulsion increases with the ambient electron temperature in the converging magnetic field topology (e.g., Damiano \& Wright, 2008; Knight, 1973; Nakamura, 2000; Rankin et al., 1999). As more electrons are effectively trapped by the mirror force as temperature increases, fewer electrons must be accelerated to higher energies to carry a similar (or in this case) smaller parallel current. This point is illustrated in Figure 5 where the net energization of electrons at the northern low-altitude boundary is presented as a function of time. The corresponding diminishment of the current can be understood by examining the profiles of the component energies in Figure 6. The drop in the energy in $u_{2}$ is indicative of ion drift energy being converted to both magnetic field and electron energy. The magnetic field energy (and correspondingly the magnitude of $j_{\|}$) drops as progressively more ion kinetic energy is transferred to electron energization to carry the parallel current as the electron temperature is increased.

As mentioned, the qualitative features of the electron energization presented in this section are very similar to that seen for toroidal modes. This similarity is to be expected since the change in wave polarization should not alter the characteristics of the electron energization which is defined by the mirror force topology rather than the polarization of the wave. The mirror force effect on $E_{\|}$in toroidal standing modes was first noted by Rankin et al. (1999; and subsequent publications, Tikhonchuk \& Rankin, 2000, 2002). Rather than in simulations as presented here, they considered the relation between $E_{\|}$and $j_{\|}$(in the quasi-static limit) via the evaluation of the nonlocal conductivity matrix derived from the low-frequency electron gyrokinetic equation (Antonsen \& Lane, 1980) in a dipolar geometry. In the next section, we extend our consideration

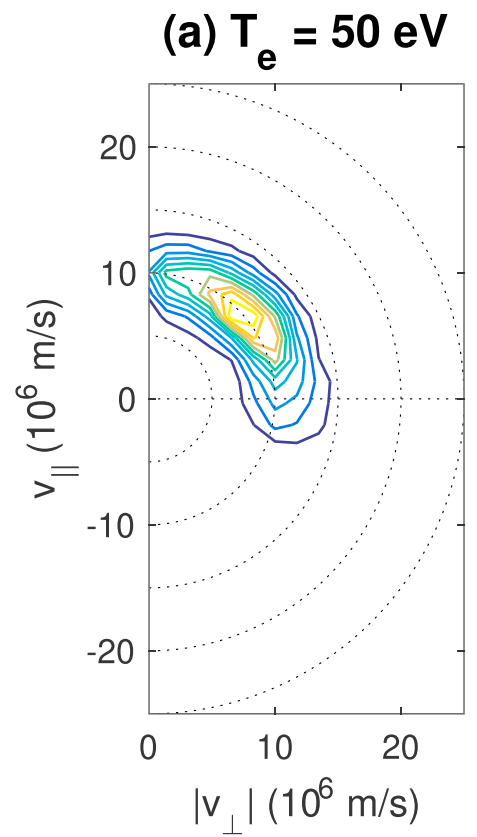

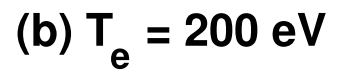

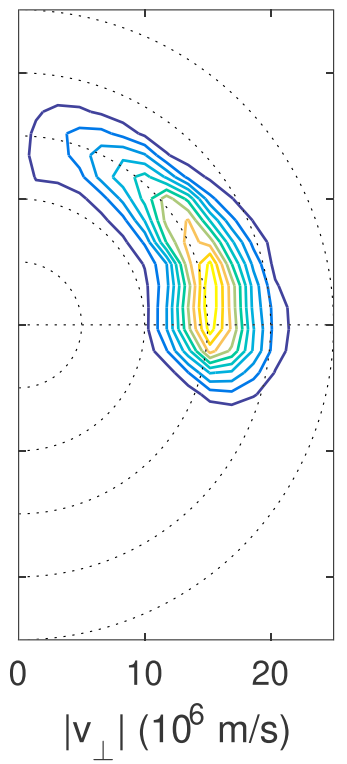

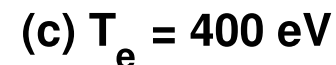

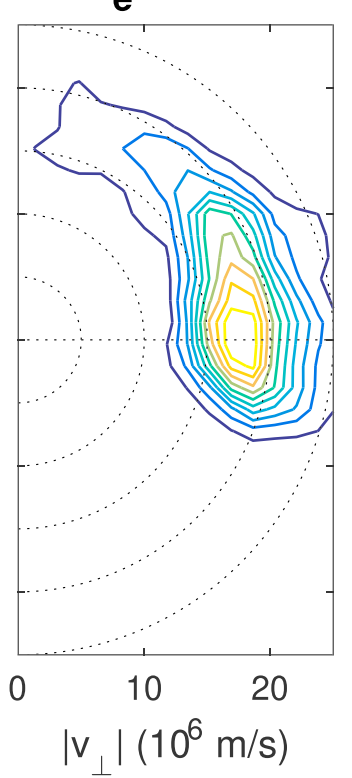

Figure 4. Distribution function at northern low-altitude boundary at $t=0.15 T_{A}$ for (a) $T_{e}=50 \mathrm{eV}$, (b) $T_{e}=200 \mathrm{eV}$, and (c) $T_{e}=400 \mathrm{eV}$. 
(a) $\mathrm{T}_{\mathrm{e}}=50 \mathrm{eV}$

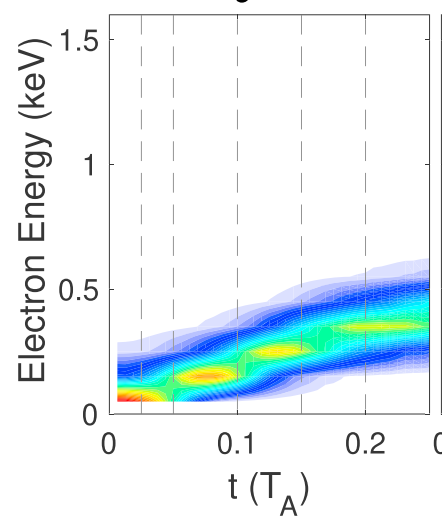

(b) $\mathrm{T}_{\mathrm{e}}=200 \mathrm{eV}$

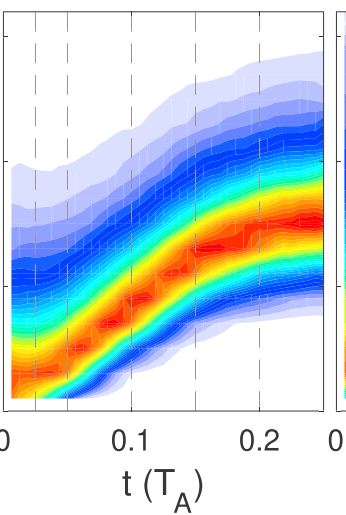

(c) $\mathrm{T}_{\mathrm{e}}=400 \mathrm{eV}$

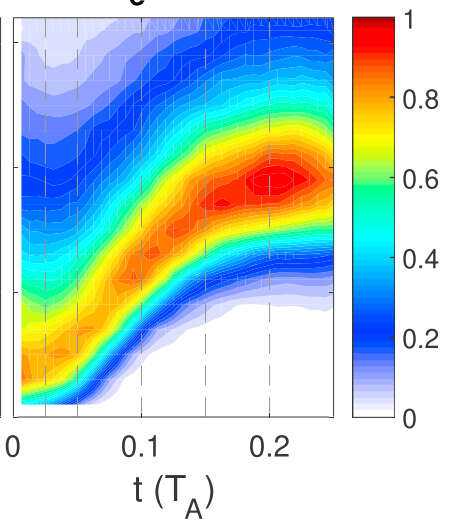

Figure 5. Energy of the accelerated electron population in the region of the current maximum as a function of time for (a) $T_{e}=50 \mathrm{eV}$, (b) $T_{e}=200 \mathrm{eV}$, and (c) $T_{e}=400 \mathrm{eV}$. In all cases, the azimuthal mode number is 250 .

of the poloidal wave system to the limit of fixed electron temperature and variable azimuthal mode number.

\subsection{Case of Fixed Electron Temperature $\left(T_{e}=200 \mathrm{eV}\right)$ and Variable $m$}

In analogy to Figure 2, Figure 7 displays the parallel current density evolution at the northern ionospheric boundary for a fixed electron temperature but for variable azimuthal mode number. Initially, the magnitude of the parallel current density increases with the azimuthal mode number (Figures 7a-7c). This trend is as would be expected because the flux tube width narrows as the azimuthal mode number is increased and the magnitude of $j_{1}$ must increase in order to carry the same total parallel current through a narrower channel. However, the increased parallel current density needs to be carried by more energetic electrons. Therefore, since the simulation is undriven, the fixed initial wave energy is dissipated most quickly in the highest azimuthal mode number case $(m=250)$ meaning that the parallel current density begins to decrease relative to the lower $m$ cases as time goes on (Figures $7 \mathrm{~d}$ and $7 \mathrm{e}$ ).

The increased energization of the electrons with the increase in $m$ (and parallel current density) is illustrated in Figures 8 and 9. As per equation (8) the increased potential drop needed to accelerate electrons results in a larger radius in velocity space (Figure 8). Figure 9 then displays the partitioning of the wave energy for the three values of the azimuthal mode number considered. The initial ion kinetic energy is consistent in each case, but as $m$ is increased, the energy transferred to the magnetic field goes down dramatically at the expense of wave energy going into the energization of precipitating electrons. This reduction in the magnetic
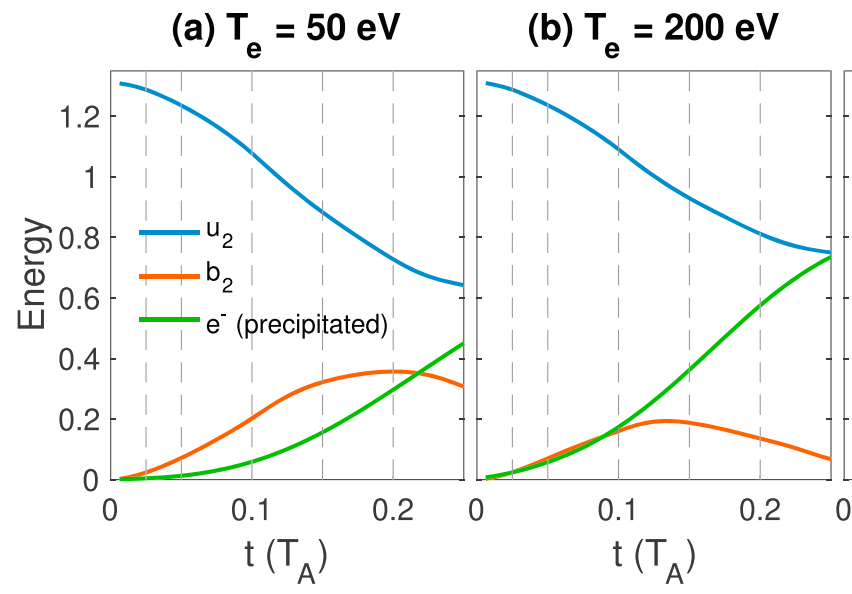

(c) $\mathrm{T}_{\mathrm{e}}=400 \mathrm{eV}$

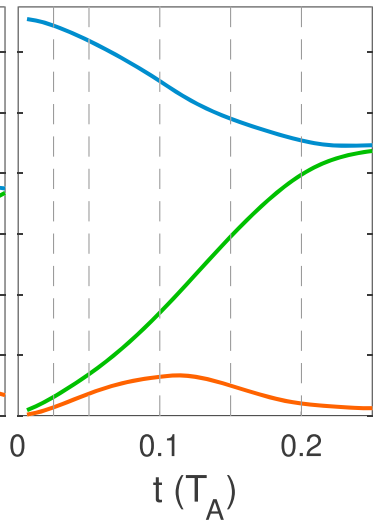

Figure 6. Component energies for (a) $T_{e}=50 \mathrm{eV}$, (b) $T_{e}=200 \mathrm{eV}$, and (c) $T_{e}=400 \mathrm{eV}$. In all cases, the azimuthal mode number is 250 . 
(a)

(b)

(c)

(d)

(e)
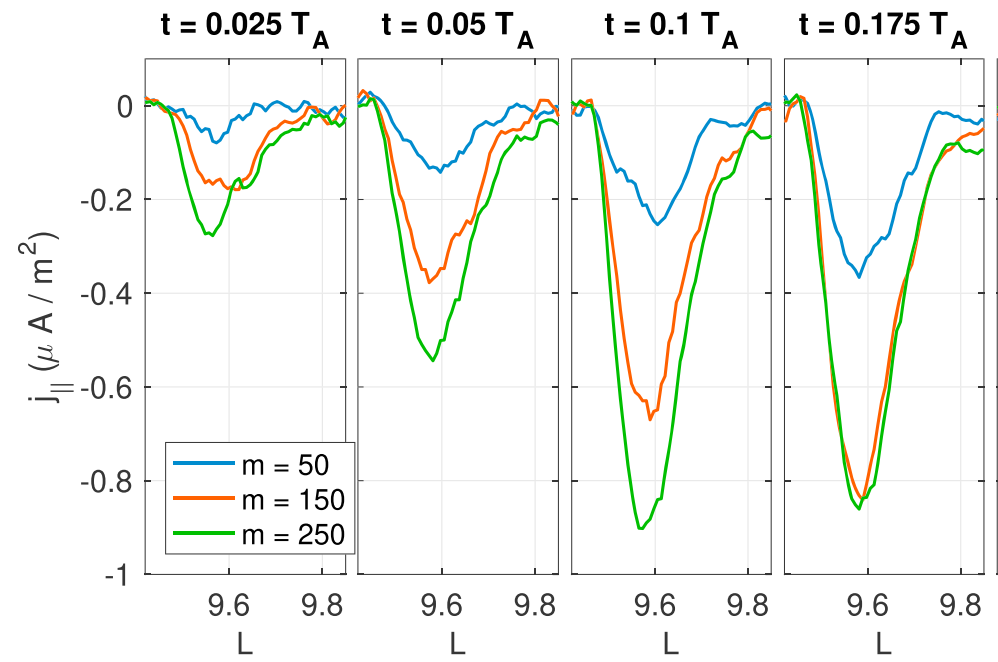

$t=0.25 T_{A}$

Figure 7. (a-e) Parallel current evolution above the northern ionospheric boundary for $T_{e}=200 \mathrm{eV}$ at the indicated times and $m$ values.

field energy also accounts for the drop in parallel current density for the largest azimuthal mode number evident in Figures 7d and 7e. It should be noted that the total energy does not appear constant in Figure 9 because the analysis neglects the initial thermal energy of the electrons.

As a final comment, it should be noted that there is one large difference between the poloidal standing mode simulations presented here and the toroidal mode studies done previously (Damiano et al., 2007; Damiano \& Wright, 2008; Damiano \& Johnson, 2012, 2013). In these latter efforts, there was substantial broadening of the parallel current profile in the radial direction, which was due to the perpendicular Poynting flux associated with the large parallel electric field $S_{2}=-E_{1} b_{3}$ (e.g., Damiano \& Johnson, 2013) where $b_{3}$ is the azimuthal magnetic field perturbation associated with the toroidal standing mode. As apparent in Figures 2 and 7, there is no similar radial broadening in the poloidal examples presented here. This lack, however, is simply because the magnetic field perturbation associated with the poloidal mode is in the radial direction
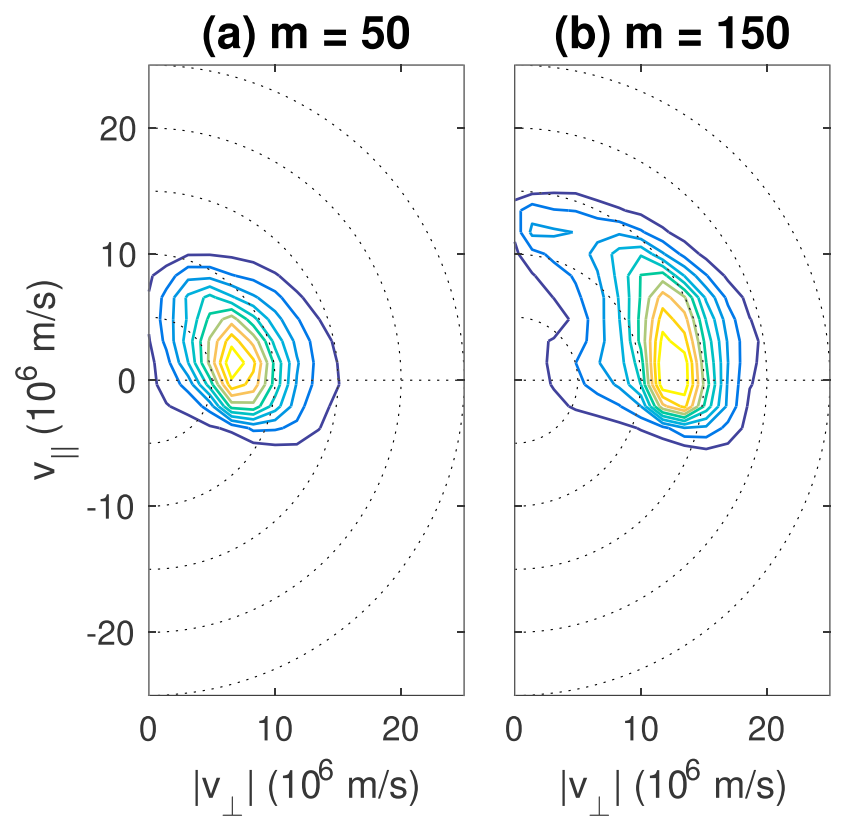
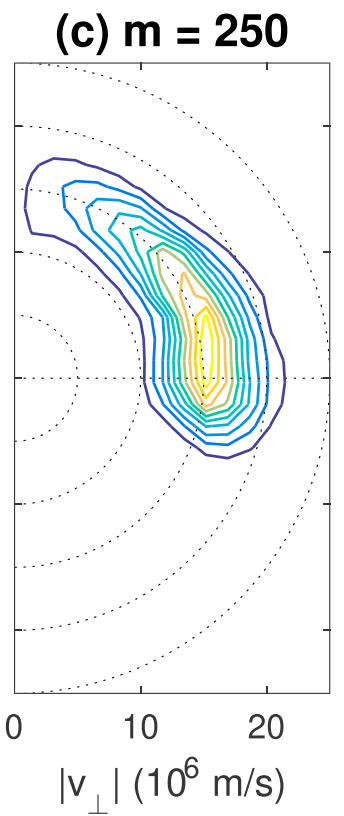

Figure 8. Distribution function at northern low-altitude boundary at $t=0.15 T_{A}$ for (a) $m=50$, (b) $m=150$, and (c) $m=250$. 


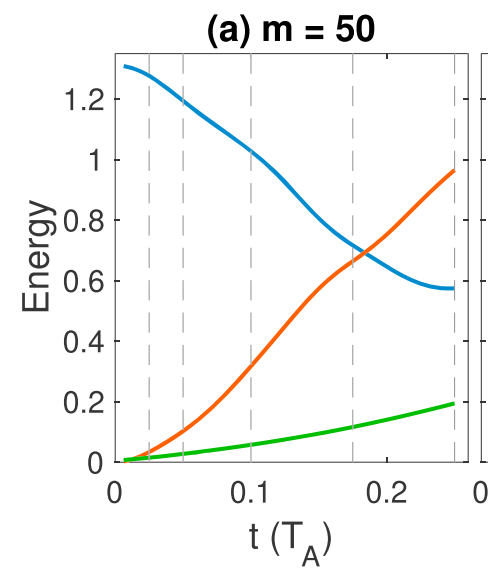

(b) $m=150$

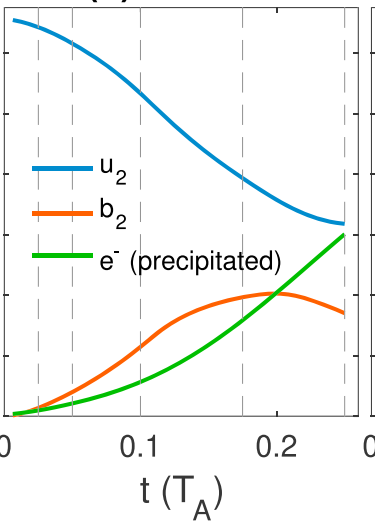

(c) $m=250$

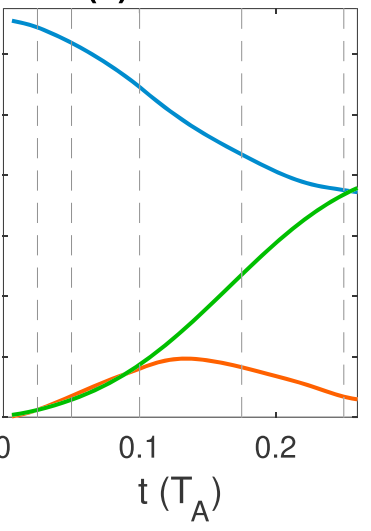

Figure 9. Component energies for (a) $m=50$, (b) $m=150 \mathrm{eV}$, and (c) $m=250 \mathrm{eV}$. In all cases, the electron temperature is $200 \mathrm{eV}$.

and the corresponding perpendicular Poynting flux would be in the azimuthal direction. This broadening is outside the remit of our present 2-D model and will be addressed when we expand to consideration of 3-D simulations. As in the 2-D case though, the effect of the broadening would further decrease the amplitude of the parallel current and electron energization to what is apparent in these examples as more of the total parallel current is being carried along adjacent field lines.

\section{Conclusions}

In this study, a 2-D hybrid gyrofluid-kinetic electron model in dipolar coordinates is used to study electron acceleration in the parallel electric fields of poloidal standing modes in the Earth's magnetosphere. It was found that, consistent with previous studies of toroidal standing modes, the electron energization increased with increasing ambient electron temperature owing to mirror force effects. The resulting simulation energized electron populations formed ring distributions in velocity space. While the electron energization increased, the net parallel current was seen to decrease because of the associated dissipation of wave energy. In the limit of fixed electron temperature, but increasing azimuthal wave number, both the parallel current and electron energization were seen to increase due to the narrowing of the flux tube in the azimuthal direction. Over the longer term though, the magnitude of the parallel current in the highest $m$ case considered decreased significantly because of the dissipation of wave energy inherent in the increased acceleration of the electrons that occurred at earlier times.

\section{References}

Antonsen, T. M., \& Lane, B. (1980). Kinetic equations for low frequency instabilities in inhomogeneous plasmas. Physics of Fluids, 23 , 1205-1214. https://doi.org/10.1063/1.863121

Chen, L., \& Hasegawa, A. (1974). A theory of long-period magnetic pulsations: 1. Steady state excitation of field line resonance. Journal of Geophysical Research, 79, 1024-1032. https://doi.org/10.1029/JA079i007p01024

Chen, L., \& Hasegawa, A. (1988). On magnetospheric hydromagnetic waves excited by energetic ring-current particles. Journal of Geophysical Research, 93, 8763-8767. https://doi.org/10.1029/JA093iA08p08763

Cheng, C. Z. (2004). Physics of substorm growth phase, onset, and dipolarization. Space Science Reviews, 113, 207-270. https://doi.org/10. 1023/B:SPAC.0000042943.59976.0e

Cheng, C. Z., \& Johnson, J. R. (1999). A kinetic-fluid model. Journal of Geophysical Research, 104, 413-428. https://doi.org/10.1029/ 1998JA900065

Cheng, C. Z., \& Lui, A. T. Y. (1998). Kinetic ballooning instability for substorm onset and current disruption observed by AMPTE/CCE. Geophysical Research Letters, 25, 4091-4094. https://doi.org/10.1029/1998GL900093

Crabtree, C., \& Chen, L. (2004). Finite gyroradius theory of drift compressional modes. Geophysical Research Letters, 31, L17804. https:// doi.org/10.1029/2004GL020660

Crabtree, C., Horton, W., Wong, H. V., \& van Dam, J (2003). Bounce-averaged stability of compressional modes in geotail flux tubes. Journal of Geophysical Research, 108(A2), 1084. https://doi.org/10.1029/2002JA009555

Dai, L., Takahashi, K., Lysak, R., Wang, C., Wygant, J. R., Kletzing, C., et al. (2015). Storm time occurrence and spatial distribution of Pc4 poloidal ULF waves in the inner magnetosphere: A Van Allen Probes statistical study. Journal of Geophysical Research: Space Physics, 120, 4748-4762. https://doi.org/10.1002/2015JA021134

Damiano, P. A., \& Johnson, J. R. (2012). Electron acceleration in a geomagnetic field line resonance. Geophysical Research Letters, 39, L02102. https://doi.org/10.1029/2011GL050264 
Damiano, P. A., \& Johnson, J. R. (2013). Mirror force induced wave dispersion in Alfvén waves. Physics of Plasmas, 20(6), 62901. https:// doi.org/10.1063/1.4810788

Damiano, P. A., Johnson, J. R., \& Chaston, C. C. (2015). Ion temperature effects on magnetotail Alfvén wave propagation and electron energization. Journal of Geophysical Research: Space Physics, 120, 5623-5632. https://doi.org/10.1002/2015JA021074

Damiano, P. A., Johnson, J. R., \& Chaston, C. C. (2016). Ion gyroradius effects on particle trapping in kinetic Alfvén waves along auroral field lines. Journal of Geophysical Research: Space Physics, 121, 10,831-10,844. https://doi.org/10.1002/2016JA022566

Damiano, P. A., Sydora, R. D., \& Samson, J. C. (2003). Hybrid magnetohydrodynamic-kinetic model of standing shear Alfvén waves. Journal of Plasma Physics, 69, 277-304. https://doi.org/10.1017/S0022377803002216

Damiano, P. A., \& Wright, A. N. (2008). Electron thermal effects in standing shear Alfvén waves. Journal of Geophysical Research, 113, A09219. https://doi.org/10.1029/2008JA013087

Damiano, P. A., Wright, A. N., Sydora, R. D., \& Samson, J. C. (2005). Hybrid magentohydrodynamic-kinetic electron closure methods and shear Alfvén waves in nonuniform plasmas. Physics of Plasmas, 12, 042105. https://doi.org/10.1063/1.1862627

Damiano, P. A., Wright, A. N., Sydora, R. D., \& Samson, J. C. (2007). Energy dissipation via electron energization in standing shear Alfvén waves. Physics of Plasmas, 14, 062904. https://doi.org/10.1063/1.2744226

Donovan, E., Mende, S., Jackel, B., Frey, H., Syrjäsuo, M., Voronkov, I., et al. (2006). The THEMIS all-sky imaging array-system design and initial results from the prototype imager. Journal of Atmospheric and Solar-Terrestrial Physics, 68(13), 1472-1487.

Elkington, S. R., Hudson, M. K., \& Chan, A. A. (1999). Acceleration of relativistic electrons via drift-resonant interaction with toroidal-mode Pc-5 ULF oscillations. Geophysical Research Letters, 26, 3273-3276. https://doi.org/10.1029/1999GL003659

Elkington, S. R., Hudson, M. K., \& Chan, A. A. (2003). Resonant acceleration and diffusion of outer zone electrons in an asymmetric geomagnetic field. Journal of Geophysical Research, 108(A3), 1116. https://doi.org/10.1029/2001JA009202

Johnson, J. R., \& Cheng, C. Z. (1997). Kinetic Alfvén waves and plasma transport at the magnetopause. Geophysical Research Letters, 24, 1423-1426. https://doi.org/10.1029/97GL01333

Klimushkin, D. Y., \& Mager, P. N. (2014). The Alfvén wave parallel electric field in non-uniform space plasmas. Astronomy and Space Science, 350, 579-583. https://doi.org/10.1007/s10509-013-1774-X

Klimushkin, D., Mager, P., \& Glassmeier, K. (2004). Toroidal and poloidal Alfvén waves with arbitrary azimuthal wavenumbers in a finite pressure plasma in the Earth's magnetosphere. Annales Geophysicae, 22, 267-287. https://doi.org/10.5194/angeo-22-267-2004

Knight, S. (1973). Parallel electric fields, Planet. Space Science, 21, 741.

Leonovich, A. S., \& Mazur, V. A. (1993). A theory of transverse small-scale standing Alfvén waves in an axially symmetric magnetosphere. Planetary and Space Science, 41, 697-717. https://doi.org/10.1016/0032-0633(93)90055-7

Leonovich, A. S., \& Mazur, V. A. (1998). Standing Alfvén waves with $m \gg 1$ in an axisymmetric magnetosphere excited by a non-stationary source. Annales Geophysicae, 16, 914-920. https://doi.org/10.1007/s00585-998-0914-z

Lotko, W., Streltsov, A. V., \& Carlson, C. W. (1998). Discrete auroral arc, electrostatic shock and suprathermal electrons powered by dispersive, anonamously resistive field line resonance. Geophysical Research Letters, 25, 4449.

Mager, P. N., Klimushkin, D. Y., \& Kostarev, D. V. (2013). Drift-compressional modes generated by inverted plasma distributions in the magnetosphere. Journal of Geophysical Research: Space Physics, 118, 4915-4923. https://doi.org/10.1002/jgra.50471

Mann, I. R., \& Wright, A. N. (1995). Finite lifetimes of ideal poloidal Alfvén waves. Journal of Geophysical Research, 100, 23,677-23,686. https://doi.org/10.1029/95JA02689

Mann, I. R., Wright, A. N., \& Hood, A. W. (1997). Phase-mixing poloidal Alfvén wave polarisations. Advances in Space Research, 20, 489-492. https://doi.org/10.1016/S0273-1177(97)00716-3

Motoba, T., Hosokawa, K., Kadokura, A., \& Sato, N. (2012). Magnetic conjugacy of northern and southern auroral beads. Geophysical Research Letters, 39, L08108. https://doi.org/10.1029/2012GL051599

Nakamura, T. K. (2000). Parallel electric field of a mirror kinetic Alfvén wave. Journal of Geophysical Research, 105, 10,729-10,738. https:// doi.org/10.1029/1999JA900494

Ozeke, L. G., \& Mann, I. R. (2008). Energization of radiation belt electrons by ring current ion driven ULF waves. Journal of Geophysical Research, 113, A02201. https://doi.org/10.1029/2007JA012468

Ozeke, L. G., Mann, I. R., Murphy, K. R., Rae, I. J., Milling, D. K., Elkington, S. R., et al. (2012). ULF wave derived radiation belt radial diffusion coefficients. Journal of Geophysical Research, 117, A04222. https://doi.org/10.1029/2011JA017463

Porazik, P., \& Lin, Z. (2011a). Gyrokinetic simulation of magnetic compressional modes in general geometry. Communications in Computational Physics, 10, 899-911. https://doi.org/10.4208/cicp.241110.280111a

Porazik, P., \& Lin, Z. (2011b). Gyrokinetic particle simulation of drift-compressional modes in dipole geometry. Physics of Plasmas, 18(7), 072107. https://doi.org/10.1063/1.3605031

Radoski, H. R. (1967). Highly asymmetric MHD resonances: The guided poloidal mode. Journal of Geophysical Research, 72, 4026-4027. https://doi.org/10.1029/JZ072i015p04026

Rae, I., Mann, I., Murphy, K., Milling, D., Parent, A., Angelopoulos, V., et al. (2009). Timing and localization of ionospheric signatures associated with substorm expansion phase onset. Journal of Geophysical Research, 114, A00C09. https://doi.org/10.1029/2008JA013559

Rankin, R., Samson, J. C., \& Tikhonchuk, V. T. (1999). Parallel electric fields in dispersive shear Alfvén waves in the dipolar magnetosphere. Geophysical Research Letters, 26, 3601-3604. https://doi.org/10.1029/1999GL010715

Samson, J. C., Rankin, R., \& Tikhonchuk, V. T. (2003). Optical signatures of auroral arcs produced by field line resonances: Comparison with satellite observations and modeling. Annales Geophysicae, 21, 933.

Shprits, Y. Y., Elkington, S. R., Meredith, N. P., \& Subbotin, D. A. (2008). Review of modeling of losses and sources of relativistic electrons in the outer radiation belt I: Radial transport. Journal of Atmospheric and Solar-Terrestrial Physics, 70, 1679-1693. https://doi.org/10.1016/ j.jastp.2008.06.008

Southwood, D. J. (1974). Some features of field line resonances in the magnetosphere. Planetary and Space Science, 22, 483-491. https:// doi.org/10.1016/0032-0633(74)90078-6

Southwood, D. J. (1976). A general approach to low-frequency instability in the ring current plasma. Journal of Geophysical Research, 81 , 3340-3348. https://doi.org/10.1029/JA081i019p03340

Takahashi, K., Hartinger, M. D., Angelopoulos, V., Glassmeier, K.-H., \& Singer, H. J. (2013). Multispacecraft observations of fundamental poloidal waves without ground magnetic signatures. Journal of Geophysical Research: Space Physics, 118, 4319-4334. https://doi.org/10. $1002 /$ jgra. 50405

Taylor, J. P. H., \& Walker, A. D. M. (1984). Accurate approximate formulae for toroidal standing hydromagnetic oscillations in a dipolar geomagnetic field. Planetary and Space Science, 32, 1119-1124. https://doi.org/10.1016/0032-0633(84)90138-7

Tikhonchuk, V. T., \& Rankin, R. (2000). Electron kinetic effects in standing shear Alfvén waves in the dipolar magnetosphere. Physics of Plasmas, 7, 2630-2645. https://doi.org/10.1063/1.874105 
Tikhonchuk, V. T., \& Rankin, R. (2002). Parallel potential driven by a kinetic Alfvén wave on geomagnetic field lines. Journal of Geophysical Research, 107(A7), 1104. https://doi.org/10.1029/2001JA000231

Ukhorskiy, A. Y., Sitnov, M. I., Takahashi, K., \& Anderson, B. J. (2009). Radial transport of radiation belt electrons due to stormtime Pc5 waves. Annales Geophysicae, 27, 2173-2181. https://doi.org/10.5194/angeo-27-2173-2009

Xia, Z., Chen, L., Zheng, L., \& Chan, A. A. (2017). Eigenmode analysis of compressional poloidal modes in a self-consistent magnetic field Journal of Geophysical Research: Space Physics, 122, 10,369-10,381. https://doi.org/10.1002/2017JA024376 